\title{
RETRACTED ARTICLE: Identification of microRNAs (miR-203/miR-7) as potential markers for the early detection of lymph node metastases in patients with cervical cancer
}

\author{
Mehri Seifoleslami $^{1}$ - Maryam Khayyat Khameneie ${ }^{2} \cdot$ Fatemeh Mashayekhi $^{3}$. \\ Farnoush Sedaghati ${ }^{4} \cdot$ Katayoun Ziari $^{5} \cdot$ Kowsar Mansouri $^{6} \cdot$ Aghdas Safari $^{1}$
}

Published online: 22 October 2015

(C) International Society of Oncology and BioMarkers (ISOBM) 2016

\section{RETRACTED ARTICLE: Tumor Biol.}

DOI 10.1007/s13277-015-4265-6

This article has been retracted at the request of the Editorin-Chief, the International Society of Oncology and BioMarkers (ISOBM) and the Publisher per the Committee on Publication Ethics guidelines. The article shows evidence of irregularities in authorship and in the materials and methods during the submission process, there is strong reason to believe that the peer review process was compromised and the article is showing similarities with the following article which was submitted within a close timeframe:

Aghdas Safari, Mehri Seifoleslami, Emad Yahaghi, Farnoush Sedaghati, Maryam Khayyat Khameneie, Upregulation of

Electronic supplementary material The online version of this article (doi:10.1007/s13277-015-4265-6.) contains supplementary material, which is available to authorized users.

Aghdas Safari

safari1344@yahoo.com

1 Department of Gynecology, Khanevadeh Hospital, AJA University of Medical Sciences, Tehran, Iran

2 Department of Gynecology, Imam Reza Hospital, AJA University of Medical Sciences, Tehran, Iran

3 Critical Care Nursing Lecturer, Faculty Member of Jiroft University of Medical Sciences, Jiroft, Iran

4 Pathology Residence, Iran University of Medical Sciences, Tehran, Iran

5 Department of Pathology, Be'sat Hospital, AJA University of Medical Sciences, Tehran, Iran

6 Young Researchers and Elite Club, East Tehran Branch, Islamic Azad University, Tehran, Iran
miR-20a and miR-10a expression levels act as potential biomarkers of aggressive progression and poor prognosis in cervical cancer, Tumor Biol. (2015) First online: 01 October 2015 DOI 10.1007/s13277-015-4064-0

Date received: 17 August 2015

The retracted article was received: 16 August 2015.

As such the validity of the content of this article cannot be verified.

The online version of this article contains the full text of the retracted article as electronic supplementary material. 